ARTICLE

\title{
Elucidation of the biosynthesis of carnosic acid and its reconstitution in yeast
}

\author{
Ulschan Scheler ${ }^{1}$, Wolfgang Brandt ${ }^{2}$, Andrea Porzel ${ }^{2}$, Kathleen Rothe ${ }^{1}$, David Manzano 3,4 , Dragana Božić ${ }^{5, \dagger}$, \\ Dimitra Papaefthimiou ${ }^{5}$, Gerd Ulrich Balcke ${ }^{1}$, Anja Henning ${ }^{1}$, Swanhild Lohse ${ }^{1}$, Sylvestre Marillonnet ${ }^{1}$, \\ Angelos K. Kanellis ${ }^{5}$, Albert Ferrer ${ }^{3,4}$ \& Alain Tissier ${ }^{1}$
}

Rosemary extracts containing the phenolic diterpenes carnosic acid and its derivative carnosol are approved food additives used in an increasingly wide range of products to enhance shelf-life, thanks to their high anti-oxidant activity. We describe here the elucidation of the complete biosynthetic pathway of carnosic acid and its reconstitution in yeast cells. Cytochrome P450 oxygenases (CYP76AH22-24) from Rosmarinus officinalis and Salvia fruticosa already characterized as ferruginol synthases are also able to produce 11-hydroxyferruginol. Modelling-based mutagenesis of three amino acids in the related ferruginol synthase (CYP76AH1) from S. miltiorrhiza is sufficient to convert it to a 11-hydroxyferruginol synthase (HFS). The three sequential C20 oxidations for the conversion of 11-hydroxyferruginol to carnosic acid are catalysed by the related CYP76AK6-8. The availability of the genes for the biosynthesis of carnosic acid opens opportunities for the metabolic engineering of phenolic diterpenes, a class of compounds with potent anti-oxidant, anti-inflammatory and anti-tumour activities.

\footnotetext{
${ }^{1}$ Department of Cell and Metabolic Biology, Leibniz Institute of Plant Biochemistry, Weinberg 3, Halle 06120, Germany. ${ }^{2}$ Department of Bioorganic Chemistry, Leibniz Institute of Plant Biochemistry, Weinberg 3, Halle 06120, Germany. ${ }^{3}$ Program of Plant Metabolism and Metabolic Engineering, Centre for Research in Agricultural Genomics, Campus UAB, 08193 Bellaterra, Spain. ${ }^{4}$ Faculty of Pharmacy, Department of Biochemistry and Molecular Biology, University of Barcelona, 08028 Barcelona, Spain. ${ }^{5}$ Group of Biotechnology of Pharmaceutical Plants, Laboratory of Pharmacognosy, Department of Pharmaceutical Sciences, Aristotle University of Thessaloniki, 54124 Thessaloniki, Greece. †Present address: Institute for Biological Research 'Siniša Stanković', University of Belgrade, Bul. despota Stefana 142, 11060 Belgrade, Serbia. Correspondence and requests for materials should be addressed to A.T. (email: alain.tissier@ipb-halle.de).
} 
T erpenoid-based drugs such as taxol and artemisinin have become indispensable in the treatment of cancer and infectious diseases ${ }^{1,2}$. In addition, belonging to the large family of terpenoids are the phenolic labdane-type diterpenes carnosic acid (CA), carnosol (CO) and pisiferic acid (PA; Fig. 1) from rosemary (Rosmarinus officinalis) and various sage species (Salvia sp.). These compounds show diverse biological and chemical activities, including anti-oxidative, anti-cancer, antiinflammatory, as well as anti-microbial properties, and have been proposed as preventive agents and treatments for neurodegenerative disorders ${ }^{3-9}$. However, the major use of CA and $\mathrm{CO}$ is based on their strong antioxidative properties, which make them highly relevant and approved food additives in Europe, China and Japan ${ }^{10}$. Furthermore, CA has been proposed as the precursor of a whole range of diterpenoids including the tanshinones from Salvia miltiorrhiza that are being actively investigated for their anti-cancer activities ${ }^{11}$. The first committed steps in the biosynthesis of this group of diterpenes are catalysed by the terpene synthases copalyl diphosphate synthase (CPS) and a kaurene synthase-like enzyme called miltiradiene synthase (MiS). CPS and MiS cyclize geranylgeranyl diphosphate (GGPP) to copalyl diphosphate and copalyl diphosphate to miltiradiene, respectively $^{12}$ (Fig. 1). The oxidation of miltiradiene to abietatriene occurs spontaneously, but can be accelerated by exposure to ultraviolet irradiation ${ }^{13,14}$. One key intermediate in the downstream pathway is ferruginol. Several cytochrome P450 monooxygenases (CYP) of the CYP76 clan from S. miltiorrhiza, $S$. fruticosa and $R$. officinalis, which are able to convert abietatriene to ferruginol, have been characterized ${ }^{14-16}$. These enzymes are collectively called ferruginol synthases (FSs). In vitro characterization of one of these enzymes (CYP76AH4 from R. officinalis) indicated that abietatriene rather than miltiradiene is the substrate ${ }^{14}$. We show here that the previously identified FS from $S$. fruticosa and R. officinalis carry out not a single but two successive oxidations leading to the next intermediate 11-hydroxyferruginol and were renamed 11-hydroxyferruginol synthases (HFSs). Taking advantage of the similarity between these HFSs and the FS, we show that the exchange of three amino acid residues in the FS from S. miltiorrhiza by the corresponding residues in HFS is sufficient to convert the FS to a HFS. We also identify a group of related CYPs from $R$. officinalis and $S$. fruticosa with $\mathrm{C}_{20}$-oxidase $\left(\mathrm{C}_{20} \mathrm{Ox}\right)$ activity whose co-expression in yeast with the diterpene synthases and HFS leads to the production of CA, itself spontaneously oxidizing to $\mathrm{CO}$.

\section{Results}

Identification of HFSs. Using a Golden Gate modular cloning vector system developed for yeast (see Methods), we first reconstituted ferruginol biosynthesis in yeast using CYPs previously characterized as FSs, namely CYP76AH1, CYP76AH4, CYP76AH22, CYP76AH23 or CYP76AH24 (Supplementary Fig. 1 and Supplementary Table 1$)^{14-16}$. Each of these P450s was coupled with the upstream biosynthetic enzymes (CPS and MiS), the CYP reductase ATR1 (ref. 17) and a GGPP synthase (GGPPS), the latter to allow for sufficient supply of GGPP. We call this group of enzymes the core module $(\mathrm{CM})$ for the production of the diterpene precursors. To ensure efficient coexpression of all genes, we developed a library of synthetic galactose inducible promoters, which are not repressed by glucose and whose members conferring the strongest expression were selected (see Methods and Supplementary Table 2). As expected, extracts of all obtained yeast strains (Supplementary Table 3) revealed the presence of miltiradiene, abietatriene and ferruginol in gas chromatography-mass spectrometry (GC-MS) measurements (Fig. 2a and Supplementary Figs 3 and 4). However, expression of either one of CYP76AH4, CYP76AH22, CYP76AH23 or CYP76AH24 resulted in the production of a new compound, which could only be found in traces when expressing CYP76AH1 (Supplementary Figs 3-5). This product was also present in small quantities in leaf surface extracts of $R$. officinalis and $S$. fruticosa. The product was purified from yeast after shake-flask expression of CYP76AH22 with the CM (Table 1) and nuclear magnetic resonance (NMR) analysis established its identity as 11-hydroxyferruginol (Supplementary Table 4) ${ }^{18}$. Thus, CYP76AH4, CYP76AH22, CYP76AH23 and CYP76AH24, which have been reported to be FSs, are in fact able to carry out a second hydroxylation and are therefore HFSs. The activity of FS and HFSs was confirmed by in vitro assays with microsomal fractions from yeast strains expressing CYP76AH1 and CYP76AH22, respectively (Supplementary Figs 6 and 7).

During NMR analysis, we noticed increasing signals that did not belong to 11-hydroxyferruginol. To check whether these signals are due to instability of 11-hydroxyferruginol, the sample was stored at $-20^{\circ} \mathrm{C}$ for $24 \mathrm{~h}$ and analysed again. Based on these specific signals, this additional compound was identified as hydroxyferruginol quinone (Supplementary Table 4 and Supplementary Fig. 8). Consequently, 11-hydroxyferruginol spontaneously auto-oxidized to the corresponding quinone ${ }^{18}$. This was further supported by the simultaneous detection of 11-hydroxyferruginol and hydroxyferruginol quinone in all
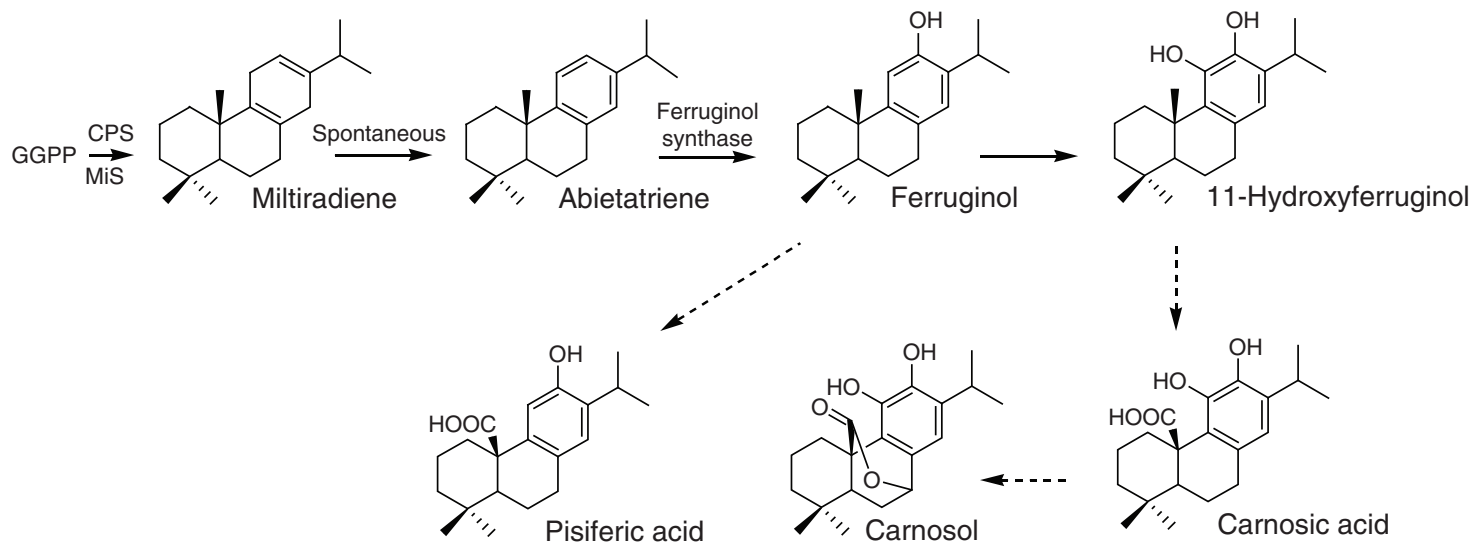

Figure 1 | Biosynthesis of PDs in Rosmarinus officinalis and sage species. State of the knowledge on the biosynthetic pathway from GGPP to CA, CO and PA before this study. The terpene synthases CPS and MiS cyclize GGPP to miltiradiene, which spontaneously oxidizes to abietatriene. Ferruginol synthases oxidize abietatriene to ferruginol. A further intermediate is presumed to be 11-hydroxyferruginol. Oxidations at the C20 position should lead to pisiferic acid (PA) from ferruginol and to carnosic acid (CA) from 11-hydroxyferruginol. Further oxidation of CA at the C7 position results in carnosol. 
yeast strains expressing the various HFSs (Supplementary Fig. 3). Such redox conversions of the catechol group to a quinone have been shown for CA and its derivatives and are thought to be the main reason for their high antioxidant activity ${ }^{4,19-23}$.
Triple-mutants of FS recapitulate HFS activity. To analyse the functional differences between FS and HFS, we applied homology modelling to CYP76AH1, CYP76AH4, CYP76AH22, CYP76AH23 and CYP76AH24. The high degree of sequence a

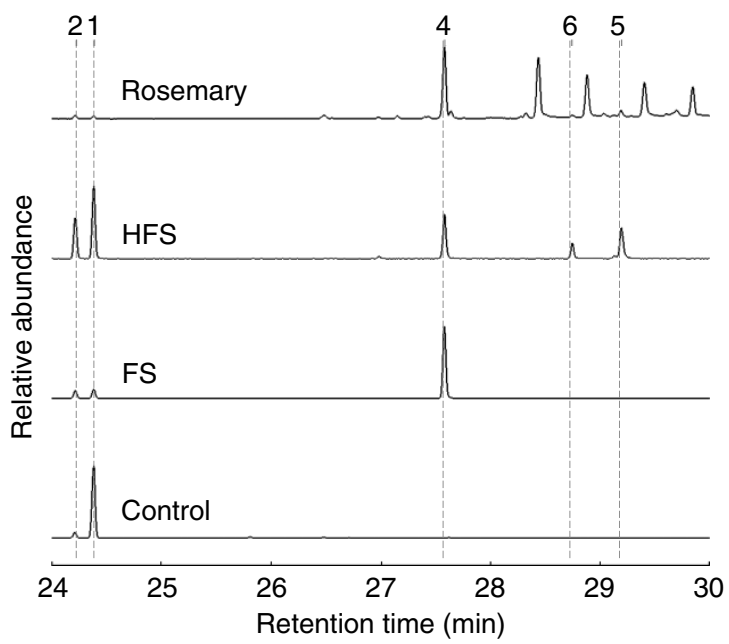

C

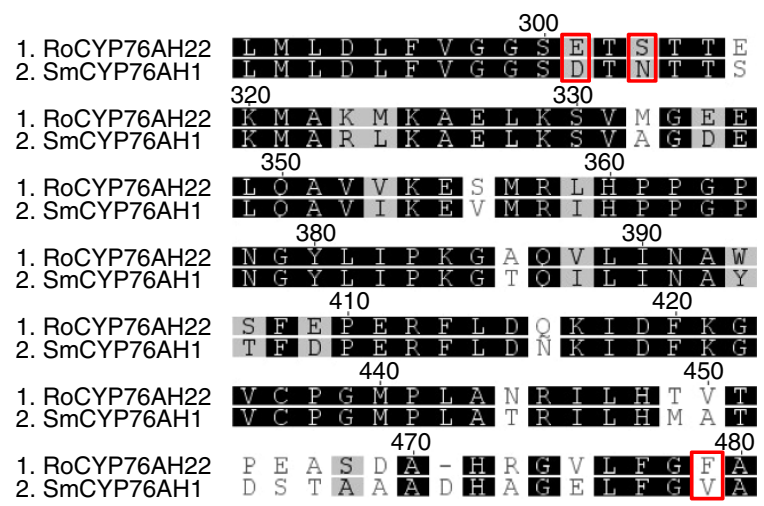

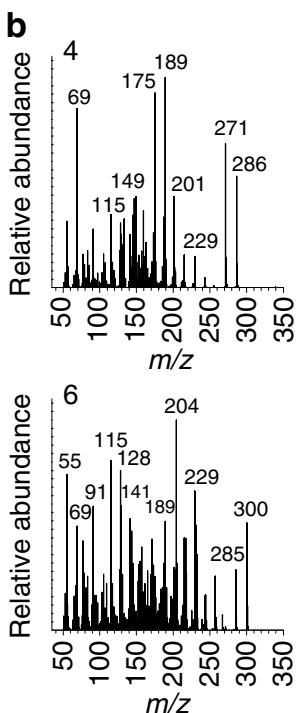

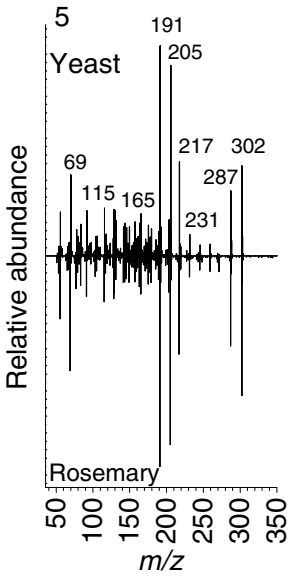

d

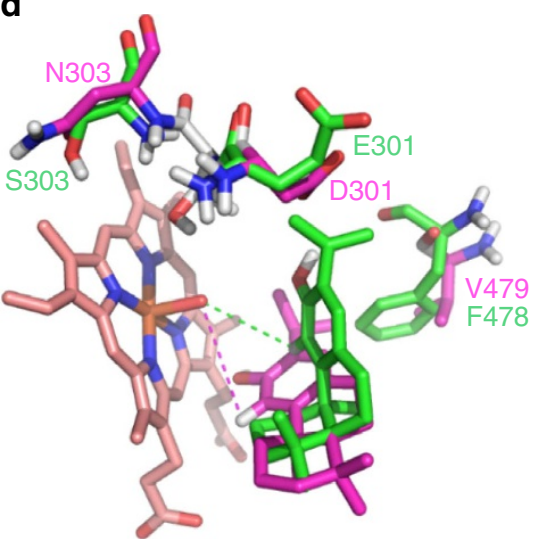



$\mathbf{f}$

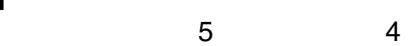

4
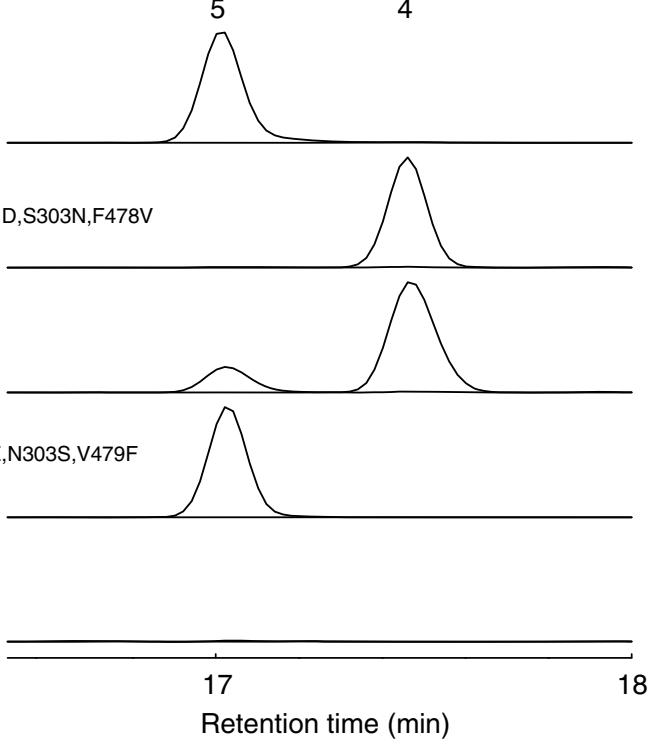
identity between these proteins (on average 80\%) resulted in predicted tertiary structures that were almost identical (see Supplementary Fig. 9 as an example). These models allowed us to identify the amino acid residues that are likely to be involved in the binding of the substrates abietatriene or ferruginol. In all enzymes the docking arrangement of abietatriene supports the oxidation reaction leading to ferruginol due to the short distance between the reactive oxygen atom bound to the haem and the proton at $\mathrm{C} 12$ required for activation (Supplementary Fig. 10). Comparative analysis of the three-dimensional models led to the identification of three amino acids in the active site of HFS (E301, S303 and F478), which are different in FS (D301, N303 and V479), and might be associated with the oxidation of ferruginol (Fig. 2c,d and Supplementary Fig. 10). In addition, two amino acids of FS (A117 and S118) are located in a $\alpha$-helix, whereas in HFS the corresponding Gly-Gly sequence adopts a coil conformation. Although both residues are rather far away from the active site, the small conformational changes may also potentially influence the activity of the enzyme (Supplementary Figs 11 and 12). All reciprocal single mutations as well as several double, triple and quadruple mutant combinations were tested in yeast in vivo co-expression assays with the CM (Supplementary Figs 13 and 14). Mutations at position 117 and 118 in HFS prevented the oxidation of ferruginol, but the reciprocal mutations in FS did not support the oxidation of ferruginol, indicating that these residues are not directly required for the oxidation of ferruginol. In contrast, the double mutant $\mathrm{FS}_{\mathrm{D} 301 \mathrm{E}, \mathrm{N} 303 \mathrm{~S}}$ could produce hydroxyferruginol, but in smaller proportions compared with HFS. Furthermore, a triple mutant $\mathrm{FS}_{\mathrm{D} 301 \mathrm{E}, \mathrm{N} 303 \mathrm{~S}, \mathrm{~V} 479 \mathrm{~F}}$ had a profile indistinguishable from HFS (Fig. 2e,f), which was also confirmed by in vitro enzyme assays (Supplementary Figs 6 and 7). The relevance of these three amino acids in the oxidation of ferruginol is confirmed by the analysis of three-dimensional models with ferruginol docked into the active site (Fig. 2d and see Supplementary Fig. 10 for details). In the HFSs (CYP76AH4, CYP76AH22, CYP76AH23 and CYP76AH24), F478 interacts with ferruginol, allowing its correct positioning for further oxidation. In contrast, in CYP76AH1, the hydrophobic interaction between the substrate and the smaller side chain of V479 at the equivalent position combined with the repulsive interaction of the carboxylic acid moiety of D301 with the isopropyl group of ferruginol, causes the adoption of a slightly different docking

Table 1 | Quantification by NMR of products formed in engineered yeast strains.

\begin{tabular}{|c|c|c|c|c|c|}
\hline Expressed enzymes & Product & $\begin{array}{c}\text { Concentration } \\
\left(\mu \mathrm{mol} \mathrm{I}^{-1}\right)\end{array}$ & $\begin{array}{c}\text { Concentration } \\
\left(m \mathrm{II}^{-1}\right)\end{array}$ & $\begin{array}{l}\text { Ratio product/ } \\
\text { miltiradiene }\end{array}$ & $\begin{array}{c}\text { Ratio product/ } \\
\text { ferruginol }\end{array}$ \\
\hline Empty vector control & ND & - & - & & \\
\hline $\begin{array}{l}\text { GGPPS:CPS:MiS:ATR1: } \\
\text { CYP76AH1 }\end{array}$ & $\begin{array}{l}\text { Miltiradiene } \\
\text { Ferruginol }\end{array}$ & $\begin{array}{l}0.93 \pm 0.18 \\
6.37 \pm 0.55\end{array}$ & $\begin{array}{l}0.25 \pm 0.05 \\
1.82 \pm 0.16\end{array}$ & $\begin{array}{c}1 \\
6.85\end{array}$ & $\begin{array}{c}0.15 \\
1\end{array}$ \\
\hline $\begin{array}{l}\text { GGPPS:CPS:MiS:ATR1: } \\
\text { CYP76AH1 D301E,N3035,V479F }\end{array}$ & $\begin{array}{l}\text { Miltiradiene } \\
\text { Ferruginol } \\
\text { 11-hydroxyferruginol }\end{array}$ & $\begin{array}{l}1.15 \pm 0.11 \\
0.41 \pm 0.09 \\
7.43 \pm 1.20\end{array}$ & $\begin{array}{l}0.31 \pm 0.03 \\
0.12 \pm 0.03 \\
2.25 \pm 0.36\end{array}$ & $\begin{array}{c}1 \\
0.35 \\
6.46\end{array}$ & $\begin{array}{c}2.80 \\
1 \\
18.12\end{array}$ \\
\hline $\begin{array}{l}\text { GGPPS:CPS:MiS:ATR1: } \\
\text { CYP76AH222 E301D,S303N,F478V }\end{array}$ & $\begin{array}{l}\text { Miltiradiene } \\
\text { Ferruginol }\end{array}$ & $\begin{array}{l}3.72 \pm 0.25 \\
6.78 \pm 0.20\end{array}$ & $\begin{array}{r}1.01 \pm 0.07 \\
1.94 \pm 0.06\end{array}$ & $\begin{array}{c}1 \\
1.82\end{array}$ & $\begin{array}{c}0.558 \\
1\end{array}$ \\
\hline $\begin{array}{l}\text { GGPPS:CPS:MiS:ATR1: } \\
\text { CYP76AH1:CYP76AK8 }\end{array}$ & $\begin{array}{l}\text { Miltiradiene } \\
\text { Ferruginol } \\
\text { Pisiferal } \\
\text { Pisiferic acid }\end{array}$ & $\begin{array}{l}2.41 \pm 0.25 \\
0.67 \pm 0.10 \\
3.03 \pm 0.84 \\
1.82 \pm 0.75\end{array}$ & $\begin{array}{l}0.66 \pm 0.07 \\
0.19 \pm 0.03 \\
0.91 \pm 0.25 \\
0.57 \pm 0.24\end{array}$ & $\begin{array}{c}1 \\
0.28 \\
1.25 \\
0.76\end{array}$ & $\begin{array}{l}3.60 \\
1 \\
4.52 \\
2.72\end{array}$ \\
\hline
\end{tabular}

CPS, copalyl diphosphate synthase; GGPPS, geranylgeranyl diphosphate synthase; MS, miltiradiene synthase; ND, not detected.

The concentrations of products, which could be detected with a signal sufficient for quantification, are given with their s.d. $(n=3)$. The concentrations are normalized to the cell density as measured by $\mathrm{OD}_{600}$. The ratios product/miltiradiene or product/ferruginol were calculated with the molar concentrations.

\footnotetext{
Figure 2 | Homology modelling-based mutagenesis of FS and HFS. (a) Part of the GC-MS profile of hexane extracts from rosemary leaf surfaces and from yeast strains co-expressing GGPPS, CPS, MS, ATR1 and indicated CYPs (selected $\mathrm{m} / \mathrm{z}$ signals: 270, 272, 286, 300 and 302). Miltiradiene (1), abietatriene (2), ferruginol (4), 11-hydroxyferruginol (5) and hydroxyferruginol quinone (6). (b) Electron impact mass spectra of ferruginol (4), 11-hydroxyferruginol (5), which was extracted from rosemary or yeast cultures, and of hydroxyferruginol quinone (6). (c) Excerpts of the aligned amino acid sequences of CYP76AH1 and CYP76AH22 with the residues that were mutagenized indicated by red rectangles. (d) Models of the active site of CYP76AH22 (green) and $\mathrm{CYP76AH} 1$ (magenta) with bound ferruginol and haem (orange carbon atoms). The models were generated using 2 $\mathrm{HI} 4$ (structure of human $\mathrm{CYP} 1 \mathrm{~A} 2$ ) as a template. Only in the case of CYP76AH22 can the hydrogen atom at $\mathrm{C} 11$ be abstracted by the reactive oxygen atom, thanks to the short distance of $3.1 \AA$ (green dashed line). In contrast, the corresponding distance in CYP76AH1 is $4.6 \AA$ (magenta dashed line), which is too large to support oxidation at this position. (e) GC-MS profile (selected $\mathrm{m} / \mathrm{z}$ signals: 286,300 and 302 ) and (f) LC-MS profile (selected $\mathrm{m} / \mathrm{z}$ signals: 285.221 and 301.217 ) of yeast strains expressing GGPPS, CPS, MS, ATR1 and indicated CYP enzymes (wild type or mutagenized). The region of the chromatograms with signals for ferruginol (4) and 11-hydroxyferruginol (5) is shown.
} 
arrangement. In this configuration, the hydrogen atom at $\mathrm{C} 11$ of ferruginol is located $4.6 \AA$ from the reactive oxygen, a distance too large to support oxidation (Supplementary Fig. 10c). Strikingly, the carboxylic moiety of E301 in HFS is involved in a salt bridge with R481 and is thus preventing the repulsive interaction with the isopropyl group of ferruginol. On the contrary, this positioning of E301 favours hydrophobic interactions via the ethylene moiety of its side chain with the isopropyl group of ferruginol. Both exchanges combined, V479/F478 and $\mathrm{D} 301 \mathrm{E}$, cause a slightly different docking orientation of ferruginol in HFS with a short distance $(3.1 \AA)$ of the substrate C11 to the reactive oxygen atom bound to the heme, thereby allowing the catalytic oxidation. These two exchanges (D301E and V479/F478) are the most important ones, whereas from the model only very minor structural alterations of the N303S substitution to the docking poses and therefore to the related catalysis can be observed.

Altogether, our results point to the essential contribution of amino acid residues at positions 301, 303 and 478 for the 11-hydroxylation of ferruginol in HFS and, remarkably, the reciprocal exchange of the corresponding residues in FS recapitulated the HFS activity. Interestingly, $\mathrm{CYP} 76 \mathrm{AH} 3$, an enzyme from $S$. miltiorrhiza that was recently shown to hydroxylate ferruginol at $\mathrm{C} 11$, has the same residues at these three positions as the HFS identified here ${ }^{24}$.

$\mathrm{C}_{20}$ oxidation by CYP76AK6-8 leads to CA. To identify the missing steps in the pathway to $\mathrm{CA}$, we searched for genes with a similar expression pattern to those of the upstream steps, namely CPS and MiS, which are preferentially expressed in trichomes on young leaves 12,16 . We focused on CYP-encoding genes, as those are frequently involved in the oxidation of terpenes ${ }^{25}$. Real-time PCR analysis of candidate genes identified from RNA-sequencing data of $R$. officinalis and S. fruticosa was performed with RNA including isolated trichomes and leaves free from trichomes, as well as young and old leaves from $R$. officinalis and S. fruticosa. CYP genes from R. officinalis (RoCYP76AK7 and RoCYP76AK8) and S. fruticosa (SfCYP76AK6) encoding highly similar proteins (Supplementary Fig. 2) showed increased transcript levels in young leaves compared with old leaves and were expressed specifically in trichomes (Fig. 3a). Interestingly, those CYPs showed high sequence similarity to the FS and HFSs.

As the precise order of the oxidations in the CA pathway was not known, we first tested these CYPs by transient co-expression with CPS and MiS in Nicotiana benthamiana. Surface extracts of leaves expressing CPS, MiS, ATR1 and CYP76AK7 analysed by GC-MS contained a new product with a molecular mass of 286 and a base peak of 257, suggesting the presence of a diterpene with an aldehyde group (Fig. 3b,c and Supplementary Fig. 15a). Co-expression of GGPPS, CPS, MiS, ATR1 and CYP76AK6, CYP76AK7 or CYP76AK8 in yeast led to the formation of the same compound (Supplementary Fig. 15b). After purification and NMR analysis, this product was identified as miltiradien-20-al (Supplementary Table 5), thus providing first evidence that CYP76AK6, CYP76AK7 and CYP76AK8 can oxidize miltiradiene at the $\mathrm{C} 20$ position and are thus named $\mathrm{C}_{20} \mathrm{Ox}$ (Fig. 4). Although miltiradien-20-ol is the expected intermediate, it could not be detected, indicating its complete conversion to miltiradien-20-al.

The relatively low conversion of miltiradiene to miltiradien20-al by the $\mathrm{C}_{20} \mathrm{Ox}$ enzymes suggested that ferruginol or 11-hydroxyferruginol rather than miltiradiene are the preferred substrates. We therefore co-expressed $\mathrm{C}_{20} \mathrm{Ox}$ together with HFS and the $\mathrm{CM}$ in yeast. GC-MS analysis of hexane extracts revealed a novel compound, which was also detected in leaf surface extracts of $R$. officinalis and S. fruticosa (Fig. $3 \mathrm{~b}, \mathrm{c}$ and
Supplementary Fig. 16). This compound was purified from rosemary plants, analysed by NMR and identified as carnosaldehyde (Supplementary Table 6). As CYP enzymes are known to carry out multiple oxidations on terpenes, for example, in the biosynthesis of abietic acid (CYP720B1) or artemisinic acid (CYP71AV1) ${ }^{26,27}$, the possibility that the $\mathrm{C}_{20} \mathrm{Ox}$ also convert this aldehyde to a carboxylic acid was considered. However, carboxylic acids, such as CA are not detectable by GC-MS. Liquid chromatography-MS (LC-MS) analysis demonstrated that $\mathrm{CA}$ was indeed formed in our strains (Fig. 3d,e and Supplementary Figs 4 and 17) and was even converted to CO, which is in agreement with the literature ${ }^{19,21,23,28}$. Although CA has been reported to be spontaneously degraded to CA quinone or to epirosmanol, rosmanol and 7-methyl-epirosmanol upon stress conditions $s^{29,30}$, none of these compounds were detected in our experiments. In coupled in vitro assays with microsome fractions from strains expressing CYP76AH22 and CYP76AK8, complete conversion of 11-hydroxyferruginol to CA could be observed (Supplementary Figs 6 and 7). In addition, as in yeast engineering experiments, $\mathrm{CO}$ could also be detected, but not the other oxidation products normally associated with non-enzymatic oxidation of CA. Control incubations of CA with single CYP or with an empty vector control did not result in increased formation of $\mathrm{CO}$, further supporting that this conversion is non-enzymatic (Supplementary Fig. 7).

To investigate whether $\mathrm{C}_{20} \mathrm{Ox}$ can, in addition to 11-hydroxyferruginol and miltiradiene, also oxidize ferruginol, the newly identified enzymes were co-expressed with CPS, MS and FS (CYP76AH1). GC-MS analysis and comparative analysis with published data ${ }^{31}$ revealed the presence of pisiferal in low amounts, indicating that indeed ferruginol is also a substrate of the $\mathrm{C}_{20} \mathrm{Ox}$ (Fig. 3b,c and Supplementary Figs 4 and 18). As for CA, PA could be found by LC-MS measurements (Fig. 3d,e). As CYP76AH1 is able to produce traces of 11-hydroxyferruginol, CA also co-accumulated with PA, albeit at much lower levels than with HFS (Supplementary Fig. 19), whereas CO and carnosaldehyde were not detected. Interestingly, co-expression of HFS and $\mathrm{C}_{20} \mathrm{Ox}$ did not result in the formation of pisiferal and $\mathrm{PA}$, although ferruginol and 11-hydroxyferruginol are still detectable. This indicates that $\mathrm{C}_{20} \mathrm{Ox}$ preferentially uses 11-hydroxyferruginol as substrate.

In vivo quantification of pathway products by NMR spectroscopy. To evaluate the respective activities of the enzyme in vivo, we performed absolute quantification by NMR of the intermediates and products of the pathway from yeast strains expressing different enzyme combinations (Table 1 and Supplementary Figs 20 and 21). NMR was chosen as a quantification method because of the lack of appropriate standards for 11-hydroxyferruginol, the different responses of GC-MS and LC-MS to ferruginol and 11-hydroxyferruginol, and the possibility to quantify compounds even without a standard (Methods). A strain expressing only the diterpene synthases produces miltiradiene at $5.86 \pm 1.52 \mu \mathrm{moll}^{-1}$. When the CYP enzymes are co-expressed with the diterpene synthases, comparable or even slightly larger amounts of the end products are produced $\left(6.37 \pm 0.55 \mu \mathrm{moll}^{-1}\right.$ of ferruginol for FS, $4.18 \pm 0.48 \mu \mathrm{moll}^{-1}$ of 11-hydroxyferruginol for HFS and $8.26 \pm 1.64 \mu \mathrm{moll}^{-1}$ of CA for HFS $+\mathrm{C}_{20} \mathrm{Ox}$ ). Whatever the CYP enzymes that are co-expressed, miltiradiene can still be detected, albeit in significantly smaller amounts compared with the CPS/MS strain. The residual presence of miltiradiene could be explained by the extreme hydrophobicity of this compound, suggesting it could be sequestered in the membrane fraction and thereby preventing its complete turnover by CYP enzymes. In a strain expressing HFS (CYP76AH22) 
residual amounts of ferruginol are present but the concentration ratio of 11-hydroxyferruginol to ferruginol is 18.17 , indicating an almost complete conversion of ferruginol to 11-hydroxyferruginol. Interestingly, the strain expressing the triple mutant $\mathrm{FS}_{\mathrm{D} 301 \mathrm{E}, \mathrm{N} 303 \mathrm{~S}, \mathrm{~V} 479 \mathrm{~F}}$ has very similar yields and ratios, notably 11-hydroxyferruginol to ferruginol of 18.12, confirming the analysis by GC-MS and LC-MS. In the strain co-expressing the HFS and the $\mathrm{C}_{20} \mathrm{Ox}$, neither 11-hydroxyferruginol nor any of the intermediates of the $\mathrm{C} 20$ oxidation could be detected by $\mathrm{NMR}$, indicating a quantitative conversion to $\mathrm{CA}$ by $\mathrm{C}_{20} \mathrm{Ox}$.

a

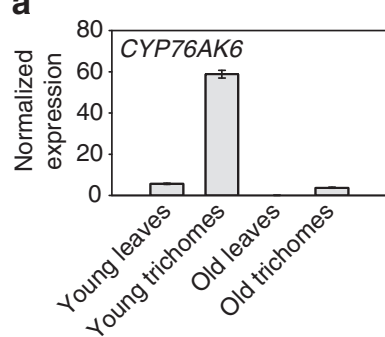

b


d

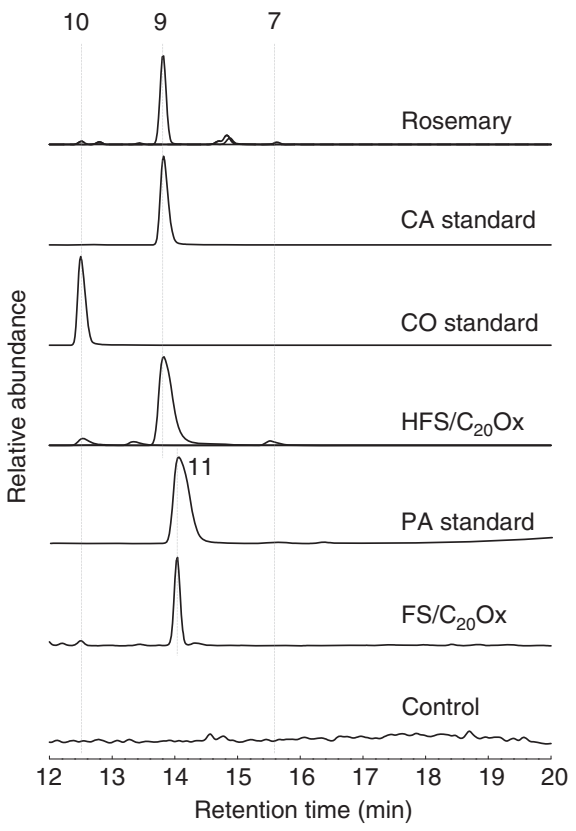

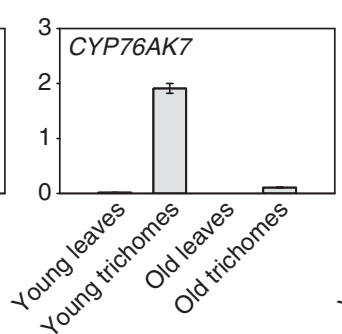

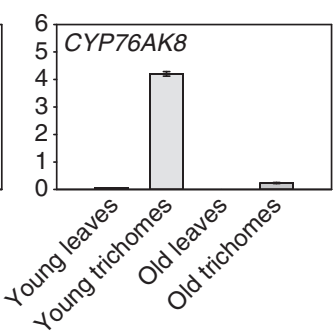

C
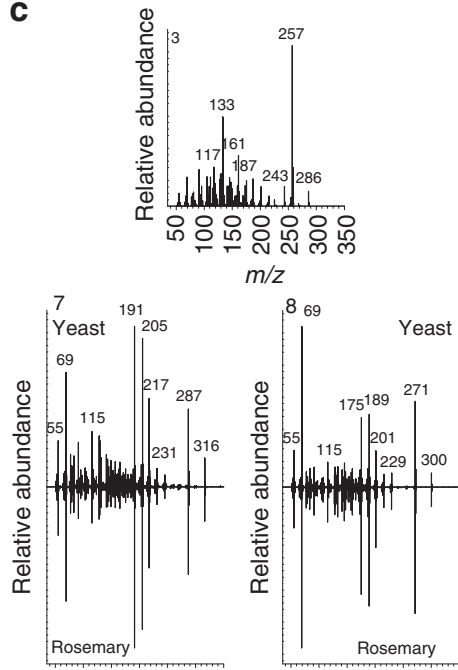

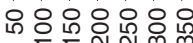

$\mathrm{m} / \mathrm{z}$

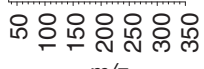

e

Furthermore, the concentration yield was the highest of all products with $8.26 \pm 1.64 \mu \mathrm{moll}^{-1}$ of CA and a ratio of CA to ferruginol of 21.18. The higher yields of CA could be explained both by the high activity of the $\mathrm{C}_{20} \mathrm{Ox}$ enzymes, but also by the higher hydrophilicity of $\mathrm{CA}$, which is more water soluble than miltiradiene or ferruginol, and is therefore less likely to be trapped in membranes. In the combination of FS with $\mathrm{C}_{20} \mathrm{Ox}$, which is not a natural combination, as the $\mathrm{C}_{20} \mathrm{Ox}$ comes from rosemary and the ferruginol synthase from $\mathrm{S}$. miltiorrhiza, the situation is different as only $1.82 \pm 0.75 \mu \mathrm{moll}^{-1}$ of PA can be produced, with a ratio of 0.76 to miltiradiene. Interestingly, higher concentrations of pisiferal, the aldehyde intermediate by $\mathrm{C}_{20}$ oxidation of ferruginol, are produced $\left(3.03 \pm 0.84 \mu \mathrm{moll}^{-1}\right)$. This supports a higher specificity of the $\mathrm{C}_{20} \mathrm{Ox}$ for 11-hydroxyferruginol compared with ferruginol. The absence of the hydroxyl group at the $\mathrm{C} 11$ position further prevents the efficient conversion of the aldehyde (pisiferal) to the end product, PA. A graphical overview of the results of phenolic diterpene pathway engineering is provided in Fig. 4.

Phylogeny of CYP76AH and CYP76AK proteins. Using the full-length amino acid sequences, a phylogenetic analysis was applied to the investigated CYP76AH and CYP76AK enzymes (Supplementary Table 7 and Supplementary Fig. 22). The resulting maximum-likelihood tree confirmed that they belong to the CYP76 clan whose members are often involved in the oxidation of terpenes ${ }^{32}$. As expected, they share the highly conserved CYP motifs, that is, the I-helix (AGxDT), K-helix (KETLR), PERF and haem-binding domain (PFGxGRRxCPG) (Supplementary Figs 1 and 2$)^{25}$. HFS and $\mathrm{C}_{20} \mathrm{Ox}$ cluster into subgroups, which form a subfamily with SmCYP76AH1, MgCYP76AH2, MgCYP76AH9 and several CYP76 members from Coleus forskohlii, the latter involved in the biosynthesis of forskolin and related compounds ${ }^{33}$. The HFSs (CYP76AH4, 22-24) constitute a clade distinct of the FS (CYP76AH1) reflecting their functional differences. Interestingly, CYP76AH1 only shares $79 \%$ sequence identity with CYP76AH24, in contrast to $90-94 \%$ between CYP76AH22-24, although both originate from sage species. Therefore, the presence of rosemary and sage sequences in the HFS clade indicates an origin predating the divergence of these species. Whether HFS evolved from FS or the opposite cannot be concluded with the current data set. The sequence similarity within the $\mathrm{C}_{20} \mathrm{Ox}$ cluster ranges from 70 to $77 \%$, whereby CYP76AK8 from $R$. officinalis is more similar to CYP76AK6 from $S$. fruticosa than to its rosemary homologue CYP76AK7. This analysis underscores the importance of the CYP76 clan in the oxidation of diterpenes, particularly in the Lamiaceae family, which is particularly rich in diverse labdanoid diterpene structures.

We note that almost identical genes were identified and characterized from S. pomifera, which when expressed in yeast also lead to production of $\mathrm{CA}^{28}$. By identifying three amino acid residues in the FS that recapitulate HFS activity and with in vivo quantification data, our results provide additional insights into the structure activity relationship of this family of CYP enzymes involved in the oxidation of diterpenes.

In conclusion, the discovery of the missing genes for the biosynthesis of CA makes it possible to engineer yeast, but also other organisms including plants, for the production at high levels of this industrially important compound. It demonstrates the power of enzymatic synthesis of high value products even compared with sophisticated chemical synthesis ${ }^{34}$. The combinatorial approach used for CA biosynthesis in yeast constitutes a versatile and efficient platform for the elucidation and production of other related terpenoids, including the promising anti-cancer tanshinones.

\section{Methods}

Plant material. R. officinalis was obtained from the Conservatoire National des Plantes à Parfum, Médicinales, Aromatiques et Industrielles (http://www.cnpmai.net/). S. fruticosa plants were kindly provided by the group of Professor Angelos Kanellis ${ }^{16}$. N. benthamiana, $R$. officinalis and $S$. fruticosa plants were grown in the greenhouse under long day conditions ( $16 \mathrm{~h}$ light $/ 8 \mathrm{~h}$ darkness) with temperatures of $25^{\circ} \mathrm{C}$ during day and $20^{\circ} \mathrm{C}$ at night, with $53 \%$ humidity. Leaf material for gene isolation and chromatographic analysis was collected in the vegetative phase.

Chemicals. Authentic standards of CA, CO and PA were purchased from Santa Cruz Biotechnology Inc., Sigma Aldrich and TCI Europe, respectively.

Tissue collection and RNA preparation for quantitative RT-PCR. Leaves from $R$. officinalis and $S$. fruticosa from Kavoussi, Crete, were collected in two developmental stages, young $(1-2 \mathrm{~cm})$ and aged $(3-4 \mathrm{~cm})$ leaves. Trichomes from leaves were isolated using dry ice ${ }^{35}$. An additional brush-abrasion step was used to remove any leftover trichomes from leaf tissue. The plant material was homogenized and RNA isolation was performed using Spectrum Plant Total RNA Kit (Sigma-Aldrich) with additional ethanol washing steps. The complementary DNA was generated from $1 \mu \mathrm{g}$ total RNA using Superscript III kit (Invitrogen) according to manufacturers' specifications and with $1 \mu \mathrm{g}$ random primers (Invitrogen).

Quantitative real-time PCR analysis. The PCR mix was prepared using KAPA SYBR FAST qPCR kit and quantitative real-time PCR analysis was performed in triplicates using the Applied Biosystems 7500 Real-Time PCR System (Invitrogen) and the following PCR conditions: $95^{\circ} \mathrm{C}$ for $2 \mathrm{~min} ; 6$ cycles of $95^{\circ} \mathrm{C}$ for $35 \mathrm{~s}, 64^{\circ} \mathrm{C}$ for $30 \mathrm{~s}, 72^{\circ} \mathrm{C}$ for $30 \mathrm{~s} ; 35$ cycles of $95^{\circ} \mathrm{C}$ for $20 \mathrm{~s}, 62^{\circ} \mathrm{C}$ for $20 \mathrm{~s}, 72^{\circ} \mathrm{C}$ for $15 \mathrm{~s}$; $72^{\circ} \mathrm{C}$ for $10 \mathrm{~min}$; plate read at $76^{\circ} \mathrm{C}$. To receive a melting curve from $70^{\circ} \mathrm{C}$ to $95^{\circ} \mathrm{C}$, data points were collected every $0.1^{\circ} \mathrm{C}$ with a $10 \mathrm{~s}$ hold between them. The data were evaluated using the delta $\mathrm{Ct} \operatorname{method}^{36}$ with the elongation factor $4 \mathrm{a}$ as reference gene.

\section{Construction of golden gate compatible yeast expression MoClo vectors.} Construction of Golden Gate compatible yeast expression vectors of level 1 and $M$ was carried out as for the MoClo system ${ }^{37,38}$. Their backbones were equipped with origins of replication for Escherichia coli (ColE1) and Saccharomyces cerevisiae ( $2 \mu \mathrm{m}$ ori) from pYES2 (Invitrogen), and in addition contained E. coli antibiotic selection markers for replication (carbenicillin in level 1 vector and spectinomycin in level $\mathrm{M}$ vector). To select for insertion into the vectors, a $L a c Z$ gene flanked by

Figure 3 | Functional analysis of $\mathbf{C}_{\mathbf{2 0}} \mathbf{O x}$. (a) Expression profile of CYP76AK6-8 in young and old trichomes and leaves without trichomes. CYP76AK6 is from S. fruticosa, CYP76AK7-8 are from $R$. officinalis. Quantitative reverse transcriptase-PCR data were obtained from three technical replicates and normalized to the eukaryotic elongation factor 4a. (b) GC-MS analysis (selected $\mathrm{m} / \mathrm{z}$ signals: 270, 272, 286, 300, 302 and 316) of extracts from rosemary leaf surfaces or from yeast strains co-expressing GGPPS, CPS, MS, ATR1, CYP76AH22 (HFS) and CYP76AK6 ( $\mathrm{C}_{20} \mathrm{Ox}$ ). The labelled peaks correspond to miltiradiene (1), abietatriene (2), ferruginol (4), 11-hydroxyferruginol (5), hydroxyferruginol quinone (6), carnosaldehyde (7) and pisiferal (8). The framed chromatogram is part of the GC-MS analysis (selected $\mathrm{m} / \mathrm{z}$ signal: 286 ) of yeast expressing GGPPS, CPS, MS, ATR1 and $\mathrm{C}_{20}$ Ox. The peak labelled (3) corresponds to miltiradien-20-al. (c) Electron impact mass spectra of PDs from yeast or rosemary. The spectra were isolated from yeast strains expressing GGPPS, CPS, MS, ATR1 and CYP76AK6 for miltiradien-20-al (3); GGPPS, CPS, MS, ATR1, CYP76AH22 and CYP76AK6 for carnosaldehyde (7); and GGPPS; CPS, MS, ATR1, CYP76AH1 and CYP76AK6 for pisiferal (8). (d) LC-MS analysis (selected m/z signals: 301.217, 315.196, 331.191 and 329.175) of rosemary extract, authentic standards and yeast extracts from strains expressing GGPPS, CPS, MS, ATR1 CYP76AH22 (HFS) and CYP76AK6 (C20Ox). Carnosaldehyde (7), CA (9), CO (10) and PA (11). (e) ESI mass spectra of PDs from yeast (expressing GGPPS, CPS, MS, ATR1, CYP76AH22 and CYP76AK6 for carnosaldehyde (7); GGPPS, CPS, MS, ATR1, CYP76AH22 and CYP76AK6 for CA (9) and CO (10); and GGPPS, CPS, MS, ATR1, CYP76AH1 and CYP76AK6 for PA (11) or rosemary and authentic standards. 


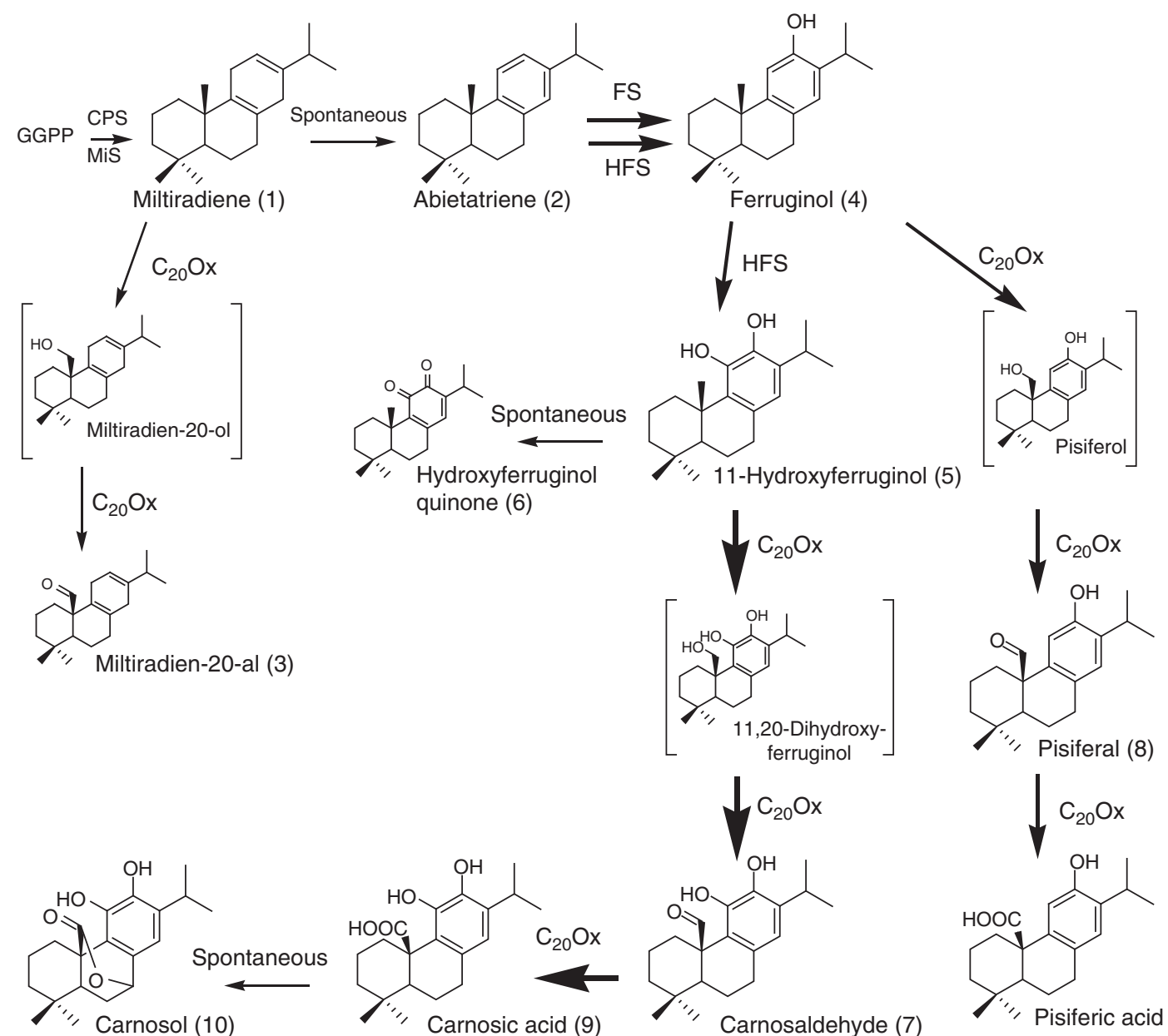


likelihood method based on the JTT/ $+\mathrm{G}$ model (five categories) ${ }^{45}$ and a bootstrap of 1,000 replicates.

Site-directed mutagenesis. Site-directed mutagenesis of Golden Gate entry vector pL0-SC containing cDNAs for CYP76AH22 or CYP76AH1 was performed using the QuikChange II Site-Directed Mutagenesis Kit (Agilent Technologies). Used primers are given in Supplementary Table 1. One microlitre of the reaction mixture was transformed into $E$. coli cells strain $\mathrm{DH} 10 \mathrm{~B}$ and plated onto agar plates containing appropriate antibiotics. Mutagenized plasmids were subsequently cloned as described above in yeast expression vectors.

Yeast microsome isolation. Microsomal preparation was done according to the literature ${ }^{40}$ with the following adaptations. The plasmids with the P450 encoding gene and ATR1 were transformed in yeast strain INVSc1 as described above. A single positive colony was used to inoculate $5 \mathrm{ml}$ of Ura-medium with $2 \%$ glucose and grown for $24 \mathrm{~h}$ at $30^{\circ} \mathrm{C}$ with shaking. The culture was then used to inoculate $100 \mathrm{ml}$ of fresh Ura-medium with $2 \%$ glucose in a $500 \mathrm{ml}$ flask, which was then further agitated at $30^{\circ} \mathrm{C}$ for $24 \mathrm{~h}$. The cells were then collected by centrifugation, resuspended in $100 \mathrm{ml}$ yeast extract-peptone-dextrose (YPD) medium with $2 \%$ galactose to induce expression and grown with shaking for $24 \mathrm{~h}$ at $30^{\circ} \mathrm{C}$. All steps were then carried out at $4{ }^{\circ} \mathrm{C}$. The cells were recovered by centrifugation and

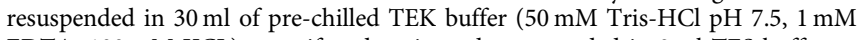
EDTA, $100 \mathrm{mM} \mathrm{KCL}$ ), centrifuged again and resuspended in $2 \mathrm{ml}$ TES buffer (50 mM Tris- $\mathrm{HCl} \mathrm{pH} \mathrm{7.5,} 600 \mathrm{mM}$ sorbitol, $10 \mathrm{gl}^{-1} \mathrm{BSA}, 1.5 \mathrm{mM} \beta$ mercaptoethanol) and transferred to a $50 \mathrm{ml}$ tube. Acid-washed autoclaved $450-600 \mu \mathrm{m}$ diameter glass beads were then added until they reached the surface of the cell suspension. The suspension was shaken vigorously by hand for $1 \mathrm{~min}$ and returned to ice for $1 \mathrm{~min}$. This was repeated four times. To wash the glass beads, $5 \mathrm{ml}$ TES buffer was added, after careful inversion the supernatant was collected and transferred to a new tube. The procedure was repeated three times. The tube was then centrifuged at $7,500 \mathrm{~g}$ for $10 \mathrm{~min}$, the supernatant transferred to ultracentrifugation tubes, which were centrifuged for $2 \mathrm{~h}$ at $100000 \mathrm{~g}$. The supernatant was discarded and gently washed successively with $5 \mathrm{ml}$ TES and $2.5 \mathrm{ml}$ TEG buffer ( $50 \mathrm{mM}$ Tris-HCl pH 7.5, $1 \mathrm{mM}$ EDTA and 30\% glycerol). The pellet was then collected with a spatula to a Potter homogenizer resuspended in $2 \mathrm{ml}$ TEG buffer and carefully homogenized. One hundred microlitre aliquots were transferred to $1.5 \mathrm{ml}$ microtubes and stored at $-80^{\circ} \mathrm{C}$ until used.

In vitro CYP assays. In a $2.0 \mathrm{ml}$ microtube, in a total volume of $600 \mu \mathrm{l}, 40 \mu \mathrm{l}$ of the microsome preparation were mixed with $100 \mu \mathrm{M}$ substrate, $1 \mathrm{mM} \mathrm{NADPH}, 50 \mathrm{mM}$ sodium phosphate $\mathrm{pH}$ and incubated at $30^{\circ} \mathrm{C}$ for $2 \mathrm{~h}$ with gentle shaking. The reaction was then extracted with $1 \mathrm{ml}$ hexane under strong agitation (vortex). After centrifugation, the organic phase was transferred to fresh tubes, dried under a $\mathrm{N}_{2}$ stream and resuspended in $50 \mu \mathrm{l}$ hexane for GC-MS analysis or $150 \mu \mathrm{l}$ methanol for LC-MS analysis.

Transient expression in $\mathbf{N}$. benthamiana. The cDNAs for CPS, MS, ATR1 and CYP76AK7 were cloned into T-DNA vectors (binary vector pL1F-1) under the control of the $35 \mathrm{~S}$ promoter and flanked by the Ocs terminator ${ }^{46}$. The resulting T-DNA plasmids were transformed into Agrobacterium tumefaciens strain GV3101::pMP90 (resistances to gentamycin and rifampicin) and streaked onto LB agar plates with appropriate antibiotics. Positively transformed colonies were inoculated into $\mathrm{LB}$ medium with selective antibiotics and grown for $18 \mathrm{~h}$ at $28^{\circ} \mathrm{C}$. To co-infiltrate several genes, appropriate volumes required to reach a final $\mathrm{OD}_{600}$ of 0.5 each were combined, centrifuged and resuspended in one-fourth volume fresh LB medium, one-fourth volume sterile water, half volume twofold concentrated infiltration medium $\left(0.3 \mathrm{M}\right.$ sucrose, $20 \mathrm{mM}$ glucose, $8.6 \mathrm{gl}^{-1}$ Murashige and Skoog medium (Duchefa Biochemie), adjusted to $\mathrm{pH}$ 5.6) and $20 \mu \mathrm{M}$ acetosyringone (diluted in dimethyl sulfoxide). The suspension was infiltrated into the abaxial leaf side of 4-week-old $N$. benthamiana plants using a syringe without needle. After treatment, the plants were cultivated under regular growth conditions for 5 days.

Homology modelling. Protein homology modelling of CYP76AH1, CYP76AH4 CYP76AH22, CYP76AH23 and CYP76AH24 was performed with YASARA ${ }^{47}$.

After search for templates in the protein database ${ }^{48}$ for each protein, 100 models were created based on alternative sequence alignments including secondary structure predictions and comparisons with ten found appropriate X-ray templates of different $\mathrm{CYPs}^{49}$. The best suited template was the X-ray structure of $2 \mathrm{HI} 4$ (refs 50,51). Models based on some other templates such as $2 \mathrm{NNJ}^{52}, 3 \mathrm{~T} 3 \mathrm{Q}^{53}, 2 \mathrm{PG} 5$ (ref. 54) and $4 \mathrm{NKY}^{55}$ are almost identical, except in some loop conformations. The substrates were docked using the molecular modelling environment programme MOE 2014.09 (https://www.chemcomp.com/). All models with docked substrates in appropriate position for an oxidation reaction in vicinity to the iron ion complexed by haem were refined with a 20 cycle simulated annealing procedure (md-refine.mcr) in YASARA. For creating the figures, the reactive oxygen atom bound to the iron ion was added manually in MOE corresponding to the proposed catalytic mechanism ${ }^{50}$. The quality of the resulting homology models were evaluated using PROCHECK ${ }^{56}$ and ProSA ${ }^{57,58}$. Despite relatively low sequence identities of the enzymes with their template, all models were of excellent quality with $>92 \%$ of the residues being in the most favoured region of the Ramachandran plot. The ProSA energy graphs were all in negative range and the calculated $z$-scores (for CYP76AH1 $=-10.84$ with 478 amino acid residues in the model and for CYP76AH22 $=-11.04$ with 481 amino acid residues) are in the range of natively folded proteins.

Production and quantification of diterpenes in yeast. Yeast expression vectors were transformed into S. cerevisiae strain INVSc1 (genotype: MATa his 3D1 leu2 trp1-289 ura3-52; Thermo Fisher Scientific) and plated out onto uracil-free selection medium $\left(1 \mathrm{gl}^{-1}\right.$ Yeast Synthetic Drop-out Medium Supplements without uracil (Sigma-Aldrich), $6.7 \mathrm{gl}^{-1}$ Yeast Nitrogen Base With Amino Acids (SigmaAldrich) and $20 \mathrm{gl}^{-1}$ Micro Agar (Duchefa Biochemie)). Positively transformed colonies were inoculated into $5 \mathrm{ml}$ YPD medium $\left(20 \mathrm{gl}^{-1}\right.$ tryptone and $10 \mathrm{gl}^{-1}$ yeast extract) containing $2 \%$ of glucose and grown for $24 \mathrm{~h}$ with shaking at $30^{\circ} \mathrm{C}$ To induce protein expression, the cell pellet was resuspended in fresh YPD medium containing $2 \%$ galactose. After another $24 \mathrm{~h}$ of cultivation, whole cultures were extracted with $2 \mathrm{ml} n$-hexane. Quantification of shake-flask expression was done according to the literature with the following adaptions ${ }^{15}$. Two single yeast colonies carrying the plasmids were inoculated into $5 \mathrm{ml}$ selection medium $\left(1 \mathrm{gl}^{-1}\right.$ Yeast Synthetic Drop-out Medium Supplements without uracil (Sigma-Aldrich) and $6.7 \mathrm{gl}^{-1}$ Yeast Nitrogen Base With Amino Acids (Sigma-Aldrich)) each. After cultivation for $24 \mathrm{~h}$, they were transferred to $50 \mathrm{ml}$ selection medium containing $2 \%$ glucose in a $250 \mathrm{ml}$ flask and grown for another $24 \mathrm{~h}$. Expression was induced the next day by resuspending the cell pellet in $50 \mathrm{ml}$ YPD medium containing $2 \%$ galactose. After cultivation for $24 \mathrm{~h}$ the optical density $\left(\mathrm{OD}_{600}\right)$ was determined and a volume with comparable cell number between all samples was used to extract the formed products with $45 \mathrm{ml}$ hexane. An aliquot was used for analysis by GC-MS and LC-MS as described below. The extracts were then evaporated to complete dryness and dissolved in $800 \mu \mathrm{C}_{6} \mathrm{D}_{6}$ containing hexamethyldisiloxane (HMDS) at a concentration of $0.0235 \mathrm{mmoll}^{-1}$ as internal standard for chemical shift referencing ( $\delta^{1} \mathrm{H}$ HMDS: 0.112 p.p.m.) as well as quantification. ${ }^{1} \mathrm{H}$ NMR spectra were recorded on a Varian/Agilent VNMRS 600 NMR spectrometer operating at a proton NMR frequency of $599.83 \mathrm{MHz}$ using a $5 \mathrm{~mm}$ inverse detection cryoprobe with the following parameters: pulse width $=6.25 \mu \mathrm{s}\left(90^{\circ}\right.$ flip angle), relaxation delay $=27.3 \mathrm{~s}$, acquisition time $=2.7 \mathrm{~s}$, number of transients $=40$ and digital resolution $0.367 \mathrm{~Hz}$ per point. An exponential multiplication of the free induction decays were performed using a line broadening factor $\mathrm{lb}=0.4 \mathrm{~Hz}$. A zero filling by a factor of 2 was used before Fourier transformation. All spectra were manually phase corrected and baseline corrected. For the quantification of metabolites, the peak area of selected proton signals belonging to the target compounds and the peak area of the internal standard (HMDS) were integrated manually. Peak assignments were done according to chemical shifts of reference compounds based on commercial standards or characterization of metabolites performed in this work.

GC-MS analysis. Plant surface extracts (R. officinalis, S. fruticosa and infiltrated $N$. benthamiana) were prepared by shaking three young leaves (R. officinalis and S. fruticosa) or ten leaf discs ( $7 \mathrm{~mm}$ diameter in case of $N$. benthamiana) with $1 \mathrm{ml}$ $n$-hexane for $2 \mathrm{~min}$ at room temperature. The extract was evaporated to complete dryness and resuspended in $200 \mu \mathrm{l} n$-hexane. The analysis of yeast and plant extracts were carried out using a Trace GC Ultra gas chromatograph (Thermo Scientific) coupled to ATAS Optic 3 injector and an ISQ single quadrupole mass spectrometer (Thermo Scientific) with electron impact ionization. Chromatographic separation was performed on a ZB-5ms capillary column $(30 \mathrm{~m} \times 0.32 \mathrm{~mm}$, Phenomenex) using splitless injection and an injection volume of $1 \mu$ l. The injection temperature rose from $60^{\circ} \mathrm{C}$ to $250^{\circ} \mathrm{C}$ with $10^{\circ} \mathrm{C} \mathrm{s}^{-1}$ and the flow rate of helium was $1 \mathrm{ml} \mathrm{min}^{-1}$. The GC oven temperature ramp was as follows: $50^{\circ} \mathrm{C}$ for $1 \mathrm{~min}, 50$ to $300^{\circ} \mathrm{C}$ with $7^{\circ} \mathrm{C} \mathrm{min}^{-1}, 300-330^{\circ} \mathrm{C}$ with $20^{\circ} \mathrm{C} \mathrm{min}^{-1}$ and $330^{\circ} \mathrm{C}$ for $5 \mathrm{~min}$. Mass spectrometry was performed at $70 \mathrm{eV}$, in a full scan mode with $\mathrm{m} / \mathrm{z}$ from 50 to 450 . Data analysis was done with the device specific software Xcalibur (Thermo Scientific).

RP-UPLC-ESI-MS/MS analysis (LC-MS). Hexane leaf surface extracts of $R$. officinalis and S. fruticosa leaves were completely evaporated under a nitrogen stream, dissolved in $150 \mu \mathrm{l}$ methanol and subjected to reversed phase-ultraperformance LC-electrospray ionization (ESI)-MS/MS analysis. Metabolites were separated using a Nucleoshell RP18 column $(2 \times 150 \mathrm{~mm}$, particle size $2.7 \mu \mathrm{m}$, Macherey-Nagel) and a ACQUITY UPLC System (Waters), including an ACQUITY Binary Solvent Manager and an ACQUITY Sample Manager $(10 \mu \mathrm{l}$ sample loop, partial loop injection mode, $5 \mu \mathrm{l}$ injection volume). For elution, aqueous $0.3 \mathrm{mmoll}^{-1} \mathrm{NH}_{4} \mathrm{HCOO}$ (adjusted to $\mathrm{pH} 3.5$ with formic acid) (A) and acetonitrile (B) were used. The elution conditions were as follows: isocratic from 0 to $2 \mathrm{~min}$ at $5 \%$ eluent B, from 2 to $19 \mathrm{~min}$ linear from 5 to $95 \%$, from 19 to $22 \mathrm{~min}$ isocratically at $95 \%$, from 22 to 22.01 min linear from 95 to $5 \%$ and from 22.01 to 24 min isocratically at $5 \%$ eluent $\mathrm{B}$. During the separation, the flow rate was set to $400 \mu \mathrm{min}^{-1}$ and the column temperature was maintained at $40^{\circ} \mathrm{C}$. A TripleToF 5600-1 mass spectrometer (AB Sciex) was used to detect the metabolites, which was 
equipped with an ESI-Duo-Turbolon-Spray interface (it operated in negative ion mode) and was controlled by Analyst 1.6 TF software (AB Sciex). The LC-ESI source operation parameters were as follows: ion spray voltage: $-4,500 \mathrm{~V}$, nebulizing gas: 60 p.s.i., source temperature: $600^{\circ} \mathrm{C}$, drying gas: 70 p.s.i., curtain gas: 35 p.s.i. Data acquisition was performed in the $\mathrm{MS}^{1}-\mathrm{ToF}_{\text {mode }} \mathrm{s}^{59}$, scanned from 250 to $500 \mathrm{Da}$ with an accumulation time of $50 \mathrm{~ms}$, and the $\mathrm{MS}^{2}$-SWATH mode, divided into $5 \mathrm{Da}$ segments of $20 \mathrm{~ms}$ accumulation time. Fifty-six separate scan experiments were carried out covering the mass range from 65 to $500 \mathrm{Da}$. In this process, a declustering potential of $35 \mathrm{~V}(-35 \mathrm{~V})$ was applied and collision energies were set to $55 \mathrm{~V}$ with a collision energy spread of $\pm 45 \mathrm{~V}$.

Isolation of new PDs and structure confirmation by NMR. For isolation of 11-hydroxyferruginol, a single yeast colony expressing GGPPS, CPS, MS, ATR1 and $\mathrm{CYP} 76 \mathrm{AH} 22$ was inoculated into $5 \mathrm{ml}$ YPD medium containing $2 \%$ glucose and grown at $28^{\circ} \mathrm{C}$ with shaking. After $24 \mathrm{~h}$, the suspension was transferred into $400 \mathrm{ml}$ culture medium for shake-flask expression and grown for another $24 \mathrm{~h}$. The expression was induced by resuspending the cell pellet in $400 \mathrm{ml}$ YPD with $2 \%$ galactose and grown as mentioned. The diterpenes were extracted from the yeast culture by adding $300 \mathrm{ml} n$-hexane and shaking thoroughly. Miltiradien-20-al was isolated from $84 \mathrm{~g}$ infiltrated leaf material of $N$. benthamiana expressing GGPPS, CPS, MS and the $\mathrm{C}_{20} \mathrm{Ox}$ CYP76AK6, which were homogenized and extracted with $800 \mathrm{ml} n$-hexane. The extract was separated from leaf material by centrifugation, evaporated and dissolved in $50 \mathrm{ml} n$-hexane. To initially separate miltiradien-20-al from other leaf compounds, the extract was applied to a SiOH column $(25 \mathrm{~g})$. Elution steps of 99:1, 98:2, 97:3 and 96:4 $n$-hexane:ethyl acetate were collected and the last fraction containing miltiradien-20-al was used for preparative high performance LC. Carnosaldehyde was extracted twice from 100 young leaves of $R$. officinalis with $80 \mathrm{ml} n$-hexane each time. The combined extracts were evaporated, dissolved in $40 \mathrm{ml}$ 95:5 $n$-hexane:ethyl acetate and applied to a $\mathrm{SiOH}$ column $(15 \mathrm{~g})$, which was equilibrate with 95:5 $n$-hexane:ethyl acetate. Carnosaldehyde eluted in the 94:6 $n$-hexane:ethyl acetate fraction which was used for further purification.

All enriched fractions were applied to a rotary evaporator and the dried products were resuspended in $1 \mathrm{ml}$ of methanol. Semi-preparative separation of 11-hydroxyferruginol and carnosaldehyde was done by LC-UV (Waters 2695 with 2996 diode array detector, Waters $\mathrm{GmbH}$ ) equipped with a fraction collector (Waters, FC III). Between the column and fraction collector, a capillary splitter (1:100) was installed which allowed ultraviolet-visible analysis at $254 \mathrm{~nm}$ and fraction collection. Using an XTerra Prep C18 column $(7.8 \mathrm{~mm} \times 150 \mathrm{~mm} \times 5 \mu \mathrm{m}$, Waters $\mathrm{GmbH}$ ) and a flow rate of $6 \mathrm{ml} \mathrm{min}^{-1}$, the same LC gradient was applied as for reverse phase-ultra-performance LC-ESI-MS/MS analysis. One hundred microlitres of each enriched fraction were injected repeatedly (four times) and a fraction eluting between 17 and $18 \mathrm{~min}$ (11-hydroxyferruginol) or between 16.1 and $16.5 \mathrm{~min}$ (carnosaldehyde) was repeatedly collected. The fractions were combined, dried down in a nitrogen stream and resuspended in deuterated solvents for NMR analysis. ${ }^{1} \mathrm{H}$ and two-dimensional spectra were recorded on an Agilent VNMRS 600 NMR spectrometer operating at $599.832 \mathrm{MHz}$ using a $5 \mathrm{~mm}$ inverse detection cryoprobe. Chemical shifts were referenced to internal tetramethylsilane $\left(\delta=0\right.$ p.p.m., $\left.{ }^{1} \mathrm{H}\right)$ or internal $\mathrm{C}_{6} \mathrm{D}_{11} \mathrm{H}\left(\delta=1.38\right.$ p.p.m., $\left.{ }^{1} \mathrm{H}\right)$ and $\mathrm{CDCl}_{3}$ $\left(\delta=77.0\right.$ p.p.m., $\left.{ }^{13} \mathrm{C}\right)$, or $\mathrm{C}_{6} \mathrm{D}_{12}\left(\delta=26.4\right.$ p.p.m., $\left.{ }^{13} \mathrm{C}\right)$ respectively.

Data availability. Data supporting the findings of this study are available within the article and its Supplementary Information files, and from the corresponding author upon reasonable request. The sequences of the CYP76AK6, CYP76AK7 and CYP76AK8 enzymes are available under GenBank accessions KX431218, KX431219 and KX431220, respectively.

\section{References}

1. Kingston, D. G. I. Taxol, a molecule for all seasons. Chem. Commun. 10, 867-880 (2001)

2. Watts, G. Nobel awarded to discoverers of ivermectin and artemisinin. BMJ 351, h5352 (2015).

3. Jordán, M. J., Lax, V., Rota, M. C., Lorán, S. \& Sotomayor, J. A. Relevance of carnosic acid, carnosol, and rosmarinic acid concentrations in the in vitro antioxidant and antimicrobial activities of Rosmarinus officinalis (L.) methanolic extracts. J. Agr. Food Chem. 60, 9603-9608 (2012).

4. Genena, A. K., Hense, H., Smânia Junior, A. \& Souza, S. M. d. Rosemary (Rosmarinus officinalis): a study of the composition, antioxidant and antimicrobial activities of extracts obtained with supercritical carbon dioxide. Ciência e Tecnologia de Alimentos 28, 463-469 (2008).

5. Poeckel, D. et al. Carnosic acid and carnosol potently inhibit human 5-lipoxygenase and suppress pro-inflammatory responses of stimulated human polymorphonuclear leukocytes. Biochem. Pharm. 76, 91-97 (2008).

6. Johnson, J. et al. Carnosol, a dietary diterpene, displays growth inhibitory effects in human prostate cancer PC3 cells leading to G2-phase cell cycle arrest and targets the $5^{\prime}$-AMP-activated protein kinase (AMPK) pathway. Pharm. Res. 25, 2125-2134 (2008).
7. Ulubelen, A., Öksüz, S., Topcu, G., Gören, A. C. \& Voelter, W. Antibacterial diterpenes from the roots of Salvia blepharochlaena. J. Nat. Prod. 64, 549-551 (2001).

8. Satoh, T. et al. Carnosic acid, a catechol-type electrophilic compound, protects neurons both in vitro and in vivo through activation of the Keap1/Nrf2 pathway via S-alkylation of targeted cysteines on Keap1. J. Neurochem. 104, 1116-1131 (2008).

9. Fischedick, J. T., Standiford, M., Johnson, D. A. \& Johnson, J. A. Structure activity relationship of phenolic diterpenes from Salvia officinalis as activators of the nuclear factor E2-related factor 2 pathway. Bioorg. Med. Chem. 21, 2618-2622 (2013).

10. Birtić, S., Dussort, P., Pierre, F. X., Bily, A. C. \& Roller, M. Carnosic acid. Phytochemistry 115, 9-19 (2015).

11. Zhang, Y. et al. Tanshinones: sources, pharmacokinetics and anti-cancer activities. Int. J. Mol. Sci. 13, 13621-13666 (2012).

12. Brückner, K. et al. Characterization of two genes for the biosynthesis of abietane-type diterpenes in rosemary (Rosmarinus officinalis) glandular trichomes. Phytochemistry 101, 52-64 (2014).

13. Brückner, K. \& Tissier, A. High-level diterpene production by transient expression in Nicotiana benthamiana. Plant Methods 9, 46 (2013).

14. Zi, J. \& Peters, R. J. Characterization of CYP76AH4 clarifies phenolic diterpenoid biosynthesis in the Lamiaceae. Org. Biomol. Chem. 11, 7650-7652 (2013).

15. Guo, J. et al. CYP76AH1 catalyzes turnover of miltiradiene in tanshinones biosynthesis and enables heterologous production of ferruginol in yeasts. Proc. Natl Acad. Sci. USA 110, 12108-12113 (2013).

16. Božić, D. et al. Towards elucidating carnosic acid biosynthesis in lamiaceae: functional characterization of the three first steps of the pathway in Salvia fruticosa and Rosmarinus officinalis. PLoS ONE 10, e0124106 (2015).

17. Mizutani, M. \& Ohta, D. Two isoforms of NADPH:cytochrome P450 reductase in Arabidopsis thaliana: gene structure, heterologous expression in insect cells, and differential regulation. Plant Physiol. 116, 357-367 (1998).

18. Tada, M., Kurabe, J., Yoshida, T., Ohkanda, T. \& Matsumoto, Y. Syntheses and antibacterial activities of diterpene catechol derivatives with abietane, totarane and podocarpane skeletons against methicillin-resistant Staphylococcus aureus and Propionibacterium acnes. Chem. Pharm. Bull. 58, 818-824 (2010).

19. Cuvelier, M. E., Berset, C. \& Richard, H. Antioxidant constituents in sage (Salvia officinalis). J. Agr. Food Chem. 42, 665-669 (1994).

20. Richheimer, S., Bernart, M., King, G., Kent, M. \& Beiley, D. Antioxidant activity of lipid-soluble phenolic diterpenes from rosemary. J. Am. Oil Chem. Soc. 73, 507-514 (1996).

21. Bicchi, C., Binello, A. \& Rubiolo, P. Determination of phenolic diterpene antioxidants in rosemary (Rosmarinus officinalis L.) with different methods of extraction and analysis. Phytochem. Anal. 11, 236-242 (2000).

22. Ho, C.-T., Wang, M., Wei, G.-J., Huang, T.-C. \& Huang, M.-T. Chemistry and antioxidative factors in rosemary and sage. Biofactors 13, 161-166 (2000).

23. Wenkert, E., Fuchs, A. \& McChesney, J. D. Chemical artifacts from the family labiatae. J. Org. Chem. 30, 2931-2934 (1965).

24. Guo, J. et al. Cytochrome $\mathrm{P} 450$ promiscuity leads to a bifurcating biosynthetic pathway for tanshinones. New Phytol. 18, 525-534 (2015).

25. Bak, S. et al. Cytochromes P450. Arabidopsis Book 9, e0144 (2011).

26. Ro, D.-K., Arimura, G.-I., Lau, S. Y. W., Piers, E. \& Bohlmann, J. Loblolly pine abietadienol/abietadienal oxidase PtAO (CYP720B1) is a multifunctional, multisubstrate cytochrome P450 monooxygenase. Proc. Natl Acad. Sci. USA 102, 8060-8065 (2005).

27. Teoh, K. H., Polichuk, D. R., Reed, D. W., Nowak, G. \& Covello, P. S. Artemisia annua L. (Asteraceae) trichome-specific cDNAs reveal CYP71AV1, a cytochrome P450 with a key role in the biosynthesis of the antimalaria sesquiterpene lactone artemisinin. FEBS Lett. 580, 1411-1416 (2006).

28. Ignea, C. et al. Carnosic acid biosynthesis elucidated by a synthetic biology platform. Proc. Natl Acad. Sci. USA 113, 3681-3686 (2016).

29. Zhang, Y. et al. Degradation study of carnosic acid, carnosol, rosmarinic acid, and rosemary extract (Rosmarinus officinalis L.) assessed using HPLC. J. Agric. Food Chem. 60, 9305-9314 (2012).

30. Schwarz, K., Ternes, W. \& Schmauderer, E. Antioxidative constituents of Rosmarinus officinalis and Salvia officinalis. III. Stability of phenolic diterpenes of rosemary extracts under thermal stress as required for technological processes. Z. Lebensm. Unters. Forch. 195, 104-107 (1992).

31. Fraga, B. M., Diaz, C. E., Guadano, A. \& Gonzalez-Coloma, A. Diterpenes from Salvia broussonetii transformed roots and their insecticidal activity. J. Agric. Food Chem. 53, 5200-5206 (2005).

32. Hofer, R. et al. Dual function of the cytochrome P450 CYP76 family from Arabidopsis thaliana in the metabolism of monoterpenols and phenylurea herbicides. Plant Physiol. 166, 1149-1161 (2014).

33. Hamberger, B., Møller, B. L., Pateraki, E., Andersen-Ranberg, J. \& Jensen, N. B. Biosynthesis of forskolin and related compounds. International patent application number WO2015/113569 A1 (2015). 
34. Jorgensen, L. et al. 14-step synthesis of $(+)$-ingenol from $(+)$-3-carene. Science 341, 878-882 (2013).

35. Yerger, E. H. et al. A rapid method for isolating glandular trichomes. Plant Physiol. 99, 1-7 (1992).

36. Livak, K. J. \& Schmittgen, T. D. Analysis of relative gene expression data using real-time quantitative PCR and the $2^{-\Delta \Delta \mathrm{CT}}$ method. Methods 25, 402-408 (2001).

37. Engler, C. \& Marillonnet, S. Golden gate cloning. Methods Mol. Biol. 1116, 119-131 (2014)

38. Werner, S., Engler, C., Weber, E., Gruetzner, R. \& Marillonnet, S. Fast track assembly of multigene constructs using Golden Gate cloning and the MoClo system. Bioeng. Bugs 3, 38-43 (2012).

39. Teo, W. S. \& Chang, M. W. Development and characterization of AND-gate dynamic controllers with a modular synthetic GAL1 core promoter in Saccharomyces cerevisiae. Biotechnol. Bioeng. 111, 144-151 (2014).

40. Urban, P., Mignotte, C., Kazmaier, M., Delorme, F. \& Pompon, D. Cloning, yeast expression, and characterization of the coupling of two distantly related Arabidopsis thaliana NADPH-cytochrome P450 reductases with P450 CYP73A5. J. Biol. Chem. 272, 19176-19186 (1997).

41. Orlova, I. et al. The small subunit of snapdragon geranyl diphosphate synthase modifies the chain length specificity of tobacco geranylgeranyl diphosphate synthase in planta. Plant Cell 21, 4002-4017 (2009).

42. Earley, K. W. et al. Gateway-compatible vectors for plant functional genomics and proteomics. Plant J. 45, 616-629 (2006).

43. Engler, C., Kandzia, R. \& Marillonnet, S. A one pot, one step, precision cloning method with high throughput capability. PLoS ONE 3, e3647 (2008).

44. Tamura, K., Stecher, G., Peterson, D., Filipski, A. \& Kumar, S. MEGA6: molecular evolutionary genetics analysis version 6.0. Mol. Biol. Evol. 30, 2725-2729 (2013).

45. Jones, D. T., Taylor, W. R. \& Thornton, J. M. The rapid generation of mutation data matrices from protein sequences. Comput. Appl. Biosci. 8, 275-282 (1992).

46. Weber, E., Gruetzner, R., Werner, S., Engler, C. \& Marillonnet, S. Assembly of designer TAL effectors by golden gate cloning. PLOS ONE 6, e19722 (2011).

47. Krieger, E. et al. Improving physical realism, stereochemistry, and side-chain accuracy in homology modeling: Four approaches that performed well in CASP8. Proteins 9, 114-122 (2009).

48. Berman, H. M. et al. The Protein Data Bank. Nucleic Acids Res. 28, 235-242 (2000).

49. Jones, D. T. Protein secondary structure prediction based on position-specific scoring matrices. J. Mol. Biol. 292, 195-202 (1999).

50. Meunier, B., de Visser, S. P. \& Shaik, S. Mechanism of oxidation reactions catalyzed by cytochrome p450 enzymes. Chem. Rev. 104, 3947-3980 (2004).

51. Sansen, S. et al. Adaptations for the oxidation of polycyclic aromatic hydrocarbons exhibited by the structure of human P450 1A2. J. Biol. Chem. 282, 14348-14355 (2007).

52. Schoch, G. A. et al. Determinants of cytochrome P450 2C8 substrate binding: structures of complexes with montelukast, troglitazone, felodipine, and 9-cisretinoic acid. J. Biol. Chem. 283, 17227-17237 (2008).

53. DeVore, N. M. et al. Structural comparison of cytochromes P450 2A6, 2A13, and 2E1 with pilocarpine. FEBS J. 279, 1621-1631 (2012).

54. Sansen, S., Hsu, M. H., Stout, C. D. \& Johnson, E. F. Structural insight into the altered substrate specificity of human cytochrome P450 2A6 mutants. Arch. Biochem. Biophys. 464, 197-206 (2007).
55. Petrunak, E. M., DeVore, N. M., Porubsky, P. R. \& Scott, E. E. Structures of human steroidogenic cytochrome P450 17A1 with substrates. J. Biol. Chem. 289, 32952-32964 (2014).

56. Laskowski, R. A., MacArthur, M. W., Moss, D. S. \& Thornton, J. M. PROCHECK: a program to check the stereochemical quality of protein structures. J. Appl. Cryst. 26, 283-291 (1993).

57. Sippl, M. J. Calculation of conformational ensembles from potentials of mean force. An approach to the knowledge-based prediction of local structures in globular proteins. J. Mol. Biol. 213, 859-883 (1990).

58. Sippl, M. J. Recognition of errors in three-dimensional structures of proteins. Proteins 17, 355-362 (1993).

59. Hopfgartner, G., Tonoli, D. \& Varesio, E. High-resolution mass spectrometry for integrated qualitative and quantitative analysis of pharmaceuticals in biological matrices. Anal. Bioanal. Chem. 402, 2587-2596 (2012).

\section{Acknowledgements}

We thank Jürgen Schmidt for assistance in the analysis of mass spectral data and Benedikt Athmer for his support concerning phylogenetic analysis. We also thank Arianne Schnabel and Sarah Vorndran for technical assistance. The work was supported in part by the TERPMED project (EC grant number 227448) and by the Leibniz Institute of Plant Biochemistry in Halle, Germany.

\section{Author contributions}

A.T., A.F. and A.K.K. conceived the project. A.T. conceived and designed the experiments. U.S., K.R., D.M., D.B., D.P., G.U.B., A.H., A.P. and W.B. performed the experiments. S.M. conceived and designed the yeast Golden gate cloning vectors. S.L. conceived, designed and made the synthetic yeast GAL-inducible promoters. U.S., A.P. and A.T. analysed the data. U.S. and A.T. wrote the paper.

\section{Additional information}

Supplementary Information accompanies this paper at http://www.nature.com/ naturecommunications

Competing financial interests: The authors declare no competing financial interests.

Reprints and permission information is available online at http://npg.nature.com/ reprintsandpermissions/

How to cite this article: Scheler, U. et al. Elucidation of the biosynthesis of carnosic acid and its reconstitution in yeast. Nat. Commun. 7, 12942 doi: 10.1038/ncomms12942 (2016)

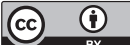

This work is licensed under a Creative Commons Attribution 4.0 International License. The images or other third party material in this article are included in the article's Creative Commons license, unless indicated otherwise in the credit line; if the material is not included under the Creative Commons license, users will need to obtain permission from the license holder to reproduce the material To view a copy of this license, visit http://creativecommons.org/licenses/by/4.0/

(C) The Author(s) 2016 